\title{
Resúmenes de Trabajos para el Concurso Académico «Dr. Mario Shapiro» 2021
}

Abstracts of Papers for the Academic Contest «Dr. Mario Shapiro» Academic Competition 2021

Resumo dos Trabalhos para o Concurso Acadêmico «Dr. Mario Shapiro» 2021

\author{
Correlación entre $\mathrm{SaO}_{2} / \mathrm{FiO}_{2}$ y $\mathrm{PaO}_{2} / \mathrm{FiO}_{2}$ en \\ pacientes bajo ventilación mecánica invasiva con \\ diagnóstico de neumonía por SARS-CoV-2 \\ Flores Benítez Gabriela, Olvera-Carranza Esteban E, \\ Guzmán-Rojas Ricardo, Palacios-Saucedo Gerardo C, \\ Ballinas-Martínez Oscar O \\ Centro Médico del Noreste, UMAE No. 25.
}

$\mathrm{La} \mathrm{PaO}_{2} / \mathrm{FiO}_{2}$ ha sido utilizada para definir el SDRA. Reportando una buena correlación entre esta y la $\mathrm{SaO}_{2} / \mathrm{FiO}_{2}$. Sin embargo, no se ha estudiado la correlación entre estos índices en población con neumonía por SARS-CoV-2. Este trabajo busca determinar la correlación de estos índices en este grupo de pacientes. Existe fuerte correlación entre la $\mathrm{SaO}_{2} / \mathrm{FiO}_{2}$ y la $\mathrm{PaO}_{2} / \mathrm{FiO}_{2}$ en pacientes con neumonía por SARS-CoV-2 bajo ventilación Mecánica.

Volumen medio plaquetario como biomarcador de respuesta inflamatoria y su utilidad como indicador temprano de mortalidad en pacientes con COVID-19 Patiño Flores Jorge Luis, Hidalgo Martínez Sandra Margarita Hospital General Regional Número 1, Centro. Hospital General Regional Número 2, El Marqués, Querétaro, Querétaro.

La sepsis es una de las enfermedades mortales más comunes. Se define como una disfunción orgánica causada por una respuesta desregulada del huésped a un proceso infeccioso determinado. Es una de las pocas condiciones médicas que ataca por igual y con gran ferocidad tanto a poblaciones en zonas de escasos recursos como a las del mundo de altos ingresos. En pacientes que cursan con un proceso inflamatorio, el aumento en la concentración de citoquinas proinflamatorias puede conducir a la liberación de plaquetas, lo que conlleva una disminución en el número de plaquetas (asociado a severidad del proceso infeccioso) e inversamente cambio en la morfología plaquetaria, aumentando así su tamaño a valores variables de volumen medio plaquetario (VMP). Está demostrado que el incremento del VMP se correlaciona con enfermedades que tienen como sustrato la inflamación, por lo que en la literatura existe evidencia de su utilidad como marcadores de inflamación, trombosis y disfunción endotelial, como lo es la sepsis. COVID-19 es una enfermedad viral emergente que fue declarada emergencia internacional el 30 de enero de 2020. Esta pandemia está provocando una movilización de la comunidad científica sin precedentes, lo que lleva asociado un número exponencialmente creciente de publicaciones en relación con la misma. Es importante determinar marcadores tempranos que ayuden a identificar de manera económica y de fácil acceso para facilitar detección y ayuden a predecir mortalidad en pacientes infectados con COVID-19. La COVID-19 se comporta como una infección compleja que puede involucrar diversos órganos, aparatos o sistemas en su anfitrión. Asimismo, diversos estudios sugieren que los perfiles hematológicos cambian durante el curso de la enfermedad del SARS-CoV-2. La producción de plaquetas aumenta a medida que disminuye el recuento de plaquetas, y un mayor número de plaquetas jóvenes implica una mayor actividad que las plaquetas menos jóvenes. De esta manera, después de la destrucción de plaquetas inducida por estrés, la disminución del conteo plaquetario estimula aún más a los megacariocitos para que produzcan una gran cantidad de plaquetas, lo que conduce a un aumento del VMP. Sin embargo, se necesitan más estudios que permitan aclarar estos mecanismos.

www.medigraphic.com/medicinacritica

\author{
Relación neutrófilos, linfocitos y plaquetas como \\ predictor de lesión renal aguda en sepsis por \\ COVID-19 en la unidad de cuidados intensivos \\ Contreras Chávez Marisol, Bravo Santibáñez Edgar, \\ Hernández González Martha Alicia, López Briones Sergio, \\ González Carrillo Pedro Luis \\ Unidad Médica de Alta Especialidad, IMSS, León Guanajuato. \\ Universidad de Guanajuato, León, Guanajuato.
}

La lesión renal aguda se encuentra en $40 \%$ de los pacientes que presentan sepsis (S-LRA), ya que la inflamación es una de las causas fisiopatológicas de la lesión renal aguda. Durante la pandemia, la principal causa de sepsis en la unidad de cuidados intensivos ( $\mathrm{UCI}$ ) fue secundaria a COVID-19, en esta se ha reportado incidencia de lesión renal de 36 a 75\%. La fisiopatología de esta complicación aún no se conoce, pero se han demostrado mecanismos similares a la lesión renal séptica típica. La relación neutrófilos, linfocitos y plaquetas (RNLP) previamente se ha asociado con la presencia de lesión renal aguda en otros ámbitos (cirugía cardiaca y cirugía abdominal mayor), y en pacientes con sepsis secundaria a COVID-19 puede ser un marcador que identifique a aquéllos con riesgo de presentar esta complicación. El objetivo principal es determinar si la relación neutrófilos, linfocitos y plaquetas es un predictor de lesión renal aguda en sepsis secundaria a COVID-19 en la UCI adultos Estudio de cohorte prospectiva, unicéntrico en mayores de 18 años que ingresen a la $\mathrm{UCI}$ con diagnóstico de sepsis por COVID-19, se realizará el cálculo de la RNLP desde el día uno hasta el día siete, se divide en dos grupos: RNLP mayor de 3 y RNLP igual o menor de 3, observando presencia o no de lesión renal aguda durante su estancia, y posterior desenlace, para el objetivo principal se hace prueba $x^{2}$, y se realiza prueba de regresión logística multivariable para la asociación de las diferentes variables con el desenlace (OR IC al 95\%). Se estudió una población de 119 pacientes, se tuvo una incidencia de S-LRA de $53.8 \%$ (IC al $95 \% 44-62 \%$ ) en casos con sepsis secundaria a COVID-19, siendo la mayoría KDIGO I (53.2\%). El grupo de RNLP mayor de 3 presentó una incidencia de $68.4 \%$ de S-LRA en comparación con el grupo de RNLP menor o igual de 3 con un $28 \%$ ( $p=0.001$, OR $4.255 \mathrm{IC}$ al $95 \%$ 1.782-10.16), los pacientes con RNLP mayor de 3 tuvieron mayor tiempo de estancia en la UCl ( 12 vs 10 días, $p=0.018$ ), y mayor tiempo de ventilación mecánica (11 vs 8 días, $p=0.003$ ). Conclusión: el incremento de la relación neutrófilos, linfocitos y plaquetas es un factor de riesgo y puede ser pronóstico para la presencia de lesión renal aguda en sepsis por COVID-19 en la UCI.

Índice neutrófilo/linfocito, volumen plaquetario medio y ancho de distribución eritrocitaria como una propuesta de puntuación predictiva en la valoración de riesgo de la paciente con preeclampsia y casos casi perdida

Pérez Piña Karla Isabel, Monares Zepeda Enrique,

Olivares Huerta Alberto

Hospital General de México.

En los últimos años, se realizaron estudios para investigar el valor diagnóstico y pronóstico del índice neutrófilo/linfocito en mujeres con preeclampsia. Lurie S, quien realizó un estudio en el Departamento de Ginecología y Obstetricia del Hospital Assaf Harofeh en Israel en 1998, describió el recuento leucocitario y la distribución diferencial en pacientes con preeclampsia y pacientes con embarazo no complicado, obteniendo como resultado que el recuento absoluto de neutrófilos fue significativamente elevado en casos con preeclampsia 
en comparación con el de los controles con embarazo no complicado $(9,410.1 \pm 3,066.9$ vs. $7,498.6 \pm 2,354.0 \times 106 / \mathrm{l}, \mathrm{p}<0.05)$. $\mathrm{El}$ objetivo principal es saber si el índice neutrófilo/linfocito, volumen plaquetario medio y ancho de distribución eritrocitaria tienen valor predictivo de preeclampsia en el «Hospital General de México» en el periodo marzo-septiembre de 2021, esto para disminuir la morbilidad y mortalidad de las pacientes gestantes. Existen escasos estudios publicados sobre la relación entre el índice neutrófilo/linfocito y preeclampsia, aunque estos han demostrado una asociación significativa; sin embargo, en nuestro medio no se ha investigado dicha asociación. Con los resultados se pretende demostrar la relación entre el índice neutrófilo/linfocito y la preeclampsia y así identificar gestantes de alto riesgo de desarrollar esta patología, de esta forma realizar un diagnóstico temprano y un tratamiento oportuno y adecuado con el fin de disminuir la morbimortalidad materna. Los valores significativamente más altos de leucocitos, neutrófilos y valores más bajos de linfocitos en la población de pacientes con preeclampsia comparado con las pacientes sanas. La media del índice neutrófilo/linfocito en casos con preeclampsia fue significativamente mayor que el grupo control $(4.7 \pm 0.24$ vs $2.67 \pm 0.17)(p<0.05)$. Al categorizar el índice neutrófilo/linfocito en dos categorías con un punto de corte de 4 y relacionarlos en función de la presencia de preeclampsia, se observó que la sensibilidad fue de $95.45 \%$ y especificidad de $98.04 \%$ en cuanto al valor predictivo positivo y valor predictivo negativo fueron $97.67 \%$ y $96.15 \%$ respectivamente; asimismo, al análisis con la curva $\mathrm{ROC}$ un valor de $\geq 4.00$ predijo la presencia de preeclampsia con una sensibilidad de $93.2 \%$ y una especificidad de $80.0 \%$ respectivamente. El índice neutrófilo/ linfocito es útil para predecir la presencia de preeclampsia en gestantes.

\section{Perfil tromboelastográfico en pacientes}

con neumonía por SARS-CoV-2

Salvador Ibarra Ibzan Jahzeel, Alva Arroyo Nancy Verónica, Ramírez Romero Eva Fabiola, Pizaña Dávila Alejandro, Huerta Escobar María Guadalupe, Gasca Aldama José Carlos Hospital Ángeles Mocel.

Introducción: La enfermedad por coronavirus 2019 (COVID-19) es una enfermedad viral causada por el síndrome agudo respiratorio severo coronavirus 2 (SARS-CoV-2). El riesgo de eventos trombóticos venosos (ETV), que aumenta en pacientes críticamente enfermos, probablemente sea aún mayor en aquéllos con SARS-CoV-2 y enfermedad crítica. Objetivos: Investigar el perfil hemostático mediante TEG en pacientes con neumonía por COVID 19. Material y métodos: Estudio observacional, retrospectivo de un solo centro hospitalario. Se inscribieron retrospectivamente pacientes hospitalizados con diagnóstico de neumonía por COVID 19, se les realizó tromboelastograma (TEG) a las 24 y 72 horas. Se realizó un análisis bivariado, para las variables cuantitativas continuas y discretas, se emplearon las pruebas de t de Student o U de Mann-Whitney. Para las variables categóricas y nominales se empleó la prueba de $\mathrm{X}^{2}$ de Pearson. Además, se realizó una curva ROC (característica de funcionamiento del receptor, por sus siglas en ingles) para determinar el punto de corte del índice de masa corporal (IMC) con mayor sensibilidad y especificidad con relación al desarrollo de alteraciones en el TEG. Posteriormente se realizó un análisis multivariado de regresión binaria logística, ajustado para las variables con significancia clínica y estadística. La significancia estadística se estableció como una $p<0.050<5 \%$. Resultados: Se incluyó un total de 66 pacientes, se observó un predominio del perfil hipercoagulable en $28(42.4 \%)$ a las 24 horas y $20(30.3 \%)$ a las 72 horas a pesar de dosis profiláctica de enoxaparina. Para determinar el punto de corte con mayor asociación entre el IMC y la presencia de trastorno en el TEG, se realizó una curva ROC, obteniendo un ABC de $64.7 \%$ ( $p=0.003$ ). Encontramos un Odds Ratio (OR) 1.8 por cada kilogramo de peso por arriba de un IMC $>26.2 \mathrm{~kg} / \mathrm{m}^{2}$, para desarrollar hipercoagulabilidad. Conclusión: El alto porcentaje de pacientes con estado hipercoagulable e hiperfibrinogenemia podría condicionar un aumento de la formación y polimerización de fibrina que puede predisponer a la trombosis. La mayoría de la población en nuestro medio cuenta con sobrepeso u obesidad, por lo que probablemente tengan necesidad de un régimen más alto de anticoagulación. Dicho esquema de tromboprofilaxis no debe ser guiado por parámetros como Dímero D, sino con una prueba más amplia del perfil hemostático como el TEG.

Supervivencia en pacientes críticamente enfermos con SARS-CoV-2 a partir de la incorporación de dexametasona al algoritmo de tratamiento López León Jorge David, González Pérez Netzahualcóyotl, Villafuerte Toral Griselda, López Carrillo Lilia, Cruz Islas Esteban Alejandro, Rosales León Jorge Roberto Centro Médico ISSEMyM Toluca.

Introducción: El tratamiento con dexametasona para pacientes con SARS-CoV-2 con lesión orgánica inflamatoria es potencialmente eficaz. Metodología: Se realizó un estudio observacional, retrospectivo, longitudinal, comparativo, en pacientes con SARS-CoV-2 con ventilación mecánica. Se calculó la probabilidad de supervivencia individual acumulada a lo largo del tiempo con el método de Kaplan-Meier en pacientes tratados con soporte multiorgánico más dexametasona vs el soporte multiorgánico estándar sin dexametasona, mediante la prueba Log Rank se identificó si existía diferencia significativa de la supervivencia entre grupos. Resultados: Se reclutaron un total de 82 pacientes con diagnóstico de SARS-CoV-2. Del total de pacientes, $43(52.4 \%)$ fueron tratados con soporte multiorgánico más dexametasona, mientras que $39(47.5 \%)$ recibieron sólo soporte multiorgánico estándar. En el seguimiento a 90 días, se presentaron en el primer grupo 11 defunciones (con una supervivencia de $74.4 \%$ ) y en el segundo grupo se presentaron 21 defunciones (con una supervivencia de 46.2\%), p 0.004. Conclusión: La administración de dexametasona a pacientes con neumonía por SARS-CoV-2, con ventilación mecánica, con una relación $\mathrm{PaO}_{2} / \mathrm{FiO}_{2}<200 \mathrm{mmHg}$, se asocia con un incremento significativo de la supervivencia a 90 días en comparación con aquéllos que no reciben este tratamiento.

De lo complejo a lo simple... Deshidrogenasa

láctica como marcador de severidad en

pacientes con infección por SARS-CoV-2

Guerrero Topete Hugo Eduardo, Gómez González Ma. Natalia,

Hernández Plata Alma Erika, Jiménez Correa Carlos

Unidad Médica de Alta Especialidad, Hospital de Especialidades

No.1, Centro Médico Nacional Bajío, Instituto Mexicano

del Seguro Social. León, Guanajuato, México.

Introducción: El estándar de referencia para el diagnóstico de COVID-19 sigue siendo la reacción en cadena de la polimerasa con transcriptasa inversa (RT-PCR) positiva, mientras que la tomografía computada (TC) simple de tórax ha sido más sensible y especifica en la clasificación de severidad. En la definición de severidad por SARSCoV-2 se han puntualizado criterios clínicos, de imagen y de laboratorio, mencionándose diversos marcadores, incluida la deshidrogenasa láctica (DHL). La DHL durante la historia ha presentado interés como un biomarcador asociado a daño pulmonar, con mayor auge durante la pandemia de influenza A H1N1, y tomada como marcador en la definición de severidad. Objetivo: Determinar el rendimiento diagnóstico de los niveles séricos de DHL al ingreso en la unidad de cuidados intensivos ( $\mathrm{UCl}$ ) como marcador de severidad en pacientes con infección por SARS-CoV-2. Métodos: Estudio observacional, analítico, longitudinal y prospectivo, llevado a cabo en dos etapas, la primera ( $1^{\circ}$ abril 2020 al 30 septiembre 2020) consistió en un estudio de correlación entre los niveles séricos de $\mathrm{DHL}$ al ingreso de $\mathrm{UCl}$ y la TC simple de tórax, se obtuvo una correlación moderada entre los niveles séricos de DHL y puntaje de severidad por tomografía pulmonar, con una $r 0.5$ y $p<0.0001$; se obtuvo un punto de corte de $\mathrm{DHL}$ sérica asociada a severidad de $396 \mathrm{U} / \mathrm{dl}(\mathrm{p}<0.001$, sensibilidad de $86.02 \%$, especificidad $75 \%$ ). La segunda etapa se extendió hasta el 31 de 
diciembre de 2020, en la cual se realiza análisis para rendimiento de pruebas diagnósticas, en la estadística inferencial se realizan pruebas de correlación, se construye un modelo de regresión lineal múltiple, se realizó análisis para pruebas diagnósticas construyendo tablas de contingencia de dos por dos, determinándose sensibilidad, especificidad, valor predictivo positivo, valor predictivo negativo, razones de verosimilitud y análisis bayesiano. Resultados: Se incluyeron 155 pacientes en el estudio para determinar el rendimiento diagnóstico de los niveles séricos de DHL en casos con infección grave por SARS-CoV-2; por análisis de regresión lineal múltiple, se determina que los niveles de $\mathrm{DHL}$ al ingreso en la $\mathrm{UCl}$ es un predictor de severidad en pacientes con SARS-CoV-2. Se obtuvo una sensibilidad de 0.92 , especificidad 0.41 , con VPP 0.854 , VPN 0.57 y una exactitud de 0.83 , un LR+ 1.57 y LR -0.63 . En los resultados de probabilidad postprueba, los pacientes con $\mathrm{DHL}>396 \mathrm{U} / \mathrm{dl}$ tienen una probabilidad de $86.3 \%$ de desarrollar cuadro severo por COVID 19. Los niveles de $\mathrm{DHL}$ tienen correlación directa con los días de estancia en la $\mathrm{UCl}$ y los días de ventilación mecánica invasiva con significancia estadística (p 0.001, p 0.33 respectivamente). Conclusión: Los niveles séricos DHL tienen una alta sensibilidad para diagnosticar severidad por COVID 19; y en ausencia de TC simple de tórax, puede predecir daño pulmonar grave. Constituye una prueba con alto poder de escrutinio en los casos severos por COVID 19; con una probabilidad de casi el doble $(L R+1.6)$ de predecir un cuadro severo.

\section{El índice de anaerobiosis predice mortalidad} en neumonía grave por SARS-CoV-2

Carballo Molina Laura, Sánchez Díaz Jesús Salvador,

Peniche Moguel Karla Gabriel, González Escudero Eduardo Alberto, Martínez Aguilar Fernando Raúl, Calyeca Sánchez María Verónica

Hospital de Especialidades No. 14, CMN «Adolfo Ruiz

Cortines", IMSS. Veracruz, Veracruz.

Introducción: Detectar y corregir el metabolismo anaerobio es indispensable en el paciente críticamente enfermo, desafortunadamente, no existe un «estándar de oro». Los pacientes con neumonía grave por SARS-CoV-2 (Severe Acute Respiratory Syndrome Coronavirus 2) presentan hipoxemia severa, aumentando el metabolismo anaerobio. El lactato y la diferencia de presión venoarterial de dióxido de carbono/diferencia del contenido arteriovenoso de oxígeno $(\Delta \mathrm{p}(\mathrm{v}-\mathrm{a})$ $\left.\mathrm{CO}_{2} / \Delta \mathrm{C}(\mathrm{a}-\mathrm{v}) \mathrm{O}_{2}\right)$ son útiles en este contexto. Material y métodos: Estudio de cohorte, prospectivo, descriptivo y analítico que incluyó pacientes ingresados a la unidad de cuidados intensivos (UCI) en el periodo comprendido entre el 18 de abril de 2020 al 18 de enero de 2021 con neumonía grave (definida por el inicio de ventilación mecánica invasiva) por SARS-CoV-2 confirmados. Resultados: En el periodo comprendido se incluyeron 91 pacientes que cumplieron con los criterios de inclusión, del total, 39 se incluyeron en el grupo de sobrevivientes y 52 en el grupo de no sobrevivientes. Se puede observar que la $\left(\Delta \mathrm{p}(\mathrm{v}-\mathrm{a}) \mathrm{CO}_{2} / \Delta \mathrm{C}(\mathrm{a}-\mathrm{v}) \mathrm{O}_{2} \mathrm{o}\right.$ índice de anaerobiosis tiene OR 4.4, IC95\% 1.51-13.04, $p=0.006$ en el análisis multivariable. Conclusión: El incremento de la $\Delta \mathrm{p}(\mathrm{v}-\mathrm{a}) \mathrm{CO}_{2} / \Delta \mathrm{C}(\mathrm{a}-\mathrm{v}) \mathrm{O}_{2} \mathrm{o}$ índice de anaerobiosis $>1.4 \mathrm{mmHg} / \mathrm{mL}$ está relacionado con 4.44 veces más riesgo de muerte en los pacientes con neumonía grave (intubados) por SARS-CoV-2.

Factores de riesgo para lesión renal aguda y

terapia de reemplazo renal en pacientes con

ventilación mecánica invasiva y COVID-19

Chávez Requena Luisa Irina, Rodríguez Zárate Cecilia, Sánchez Calzada Armando, Chaires Gutiérrez Rodrigo,

Aguirre Sánchez Janet Silvia

Centro Médico ABC.

Introducción: Desde diciembre de 2019, cuando el coronavirus respiratorio tipo 2 y el síndrome de insuficiencia respiratoria agudo (SDRA) por coronavirus tipo 2 (enfermedad por coronavirus 2019 [COVID-19]) se desarrolló en Wuhan, China, se ha convertido en una pandemia mundial, con 105,333,798 casos reportados el 4 de febrero de 2021. El 27 de febrero de 2020, la Ciudad de México reportó el primer caso de COVID-19, seguido de un crecimiento masivo de infecciones en todo el país. El número total de casos hasta hoy es de $1,886,245$ con 81,223 casos activos estimados, $18.77 \%$ de los casos han requerido hospitalización. El número total de muertes es de 164,290 con una estimación de 184,125. La lesión renal aguda (LRA) se encontró en $28 \%$ de los pacientes hospitalizados y en $46 \%$ de los casos en estado crítico, contribuyendo a una mortalidad significativamente mayor. La identificación de los factores de riesgo es importante para orientar la toma de decisiones tempranas en la clasificación de los pacientes para una monitorización más intensiva y prevenir el aumento de la mortalidad. Objetivo: Analizar el nivel de PEEP y factores inflamatorios que intervienen en el desarrollo de LRA e inicio de TRR en pacientes con COVID-19. Material y métodos: Se realizó un estudio observacional, transversal y retrolectivo en pacientes con ventilación mecánica con SARS-CoV-2 que presentaron LRA y necesidad de TRR ingresados en la unidad de cuidados intensivos respiratorios del Centro Médico ABC. Se realizó análisis estadístico de medidas de tendencia central descriptivo, para la identificación de la variable con mayor impacto para el desarrollo de LRA y terapia dialítica se realizó factor predictivo positivo, prueba Pearson para correlacionar con terapia de reemplazo renal. Conclusión: Se encontró una correlación de niveles altos de PEEP y lesión renal aguda. Los marcadores inflamatorios al ingreso del paciente (específicamente IL-6) son parámetros adecuados para guiar el tratamiento; sin embargo, también son de utilidad para orientar a un pronóstico.

Sedación con sistema AnaConDa en pacientes COVID-19 crítico y su impacto en días de ventilación mecánica

Ramírez García Héctor Alejandro, Cerda Arteaga Juana María, Chávez Pérez Carlos, Sánchez Nava Víctor Manuel Hospital Sán José Tec de Monterrey.

La sedación en el paciente crítico es esencial para asegurar un desenlace óptimo. El desabasto de medicamentos ha sido un reto frecuente durante la pandemia por COVID-19, repercutiendo principalmente en aquellos medicamentos empleados para la sedación y la analgesia. Esto ha llevado a buscar alternativas para la sedación intravenosa, siendo una de ellas la administración de anestésicos volátiles. La evidencia respecto a uso de sedación volátil en el paciente crítico apoya a su empleo en el síndrome de dificultad respiratoria aguda, pues se ha demostrado que mejora el intercambio gaseoso, reduce el edema alveolar y la inflamación pulmonar. Asimismo se ha reportado disminución del tiempo de destete de la ventilación mecánica posterior a la suspensión del medicamento. Se realizó un estudio de cohorte retrospectivo, comparativo y longitudinal en una unidad de terapia intensiva mediante la búsqueda intencionada en el expediente electrónico y el análisis de los datos de aquellos pacientes con diagnóstico de COVID-19 crítico que ameritaron el uso de ventilación mecánica e inicio de sedación intravenosa y volátil con sevoflurano durante el periodo comprendido desde el $1^{\circ}$ de enero de 2021 al 30 de junio de 2021. Estos datos se compararon con un grupo de pacientes seleccionados aleatoriamente que estuvieron internados en ese mismo periodo y que recibieron ventilación mecánica y sedación intravenosa exclusiva. Se analizó como desenlace primario si existía una diferencia significativa en el número de días de ventilación mecánica y como objetivos secundarios la presencia de diferencia significativa en los días de hospitalización, días de estancia en terapia intensiva, incidencia de neumonía intrahospitalaria y en la incidencia de mortalidad. Se identificaron 43 pacientes que recibieron sedación volátil e intravenosa, de los cuales 36 cumplían con las características necesarias para ser incluidos en el estudio. Recibieron en promedio seis días de sedación con sevoflurano. Se compararon con 42 casos que recibieron sedación intravenosa exclusiva. En el análisis poblacional se encontró una diferencia significativa de edad en la población que recibió sedación volátil. Dentro de los desenlaces estudiados se encontró una diferencia significati- 
va en los días de hospitalización, favoreciendo el empleo de sevoflurano. Ninguno de los otros desenlaces estudiados mostró diferencia significativa. La sedación volátil es una herramienta útil que puede ofrecer múltiples beneficios a los pacientes de terapia intensiva con necesidad de ventilación mecánica. El presente estudio demostró una disminución significativa del tiempo de estancia hospitalaria; sin embargo, se limita por un sesgo de edad en el subgrupo de sevoflurano. Es necesario realizar estudios con muestras más grandes, aleatorizados y con criterios estandarizados para la aplicación de la terapia con el fin de validar estos hallazgos.

Efecto del óxido nítrico inhalado en la mecánica ventilatoria y oxigenación de pacientes con hipoxemia grave refractaria a prono prolongado y bloqueo neuromuscular secundaria a infección por SARS-CoV-2 Castro Serrano Alejandro, Sánchez Calzada Armando, Aguirre Sánchez Janet Silvia, Chaires Gutiérrez Rodrigo, Hernández Sánchez Nancy

Centro Médico ABC.

El $14.6 \%$ de los pacientes infectados por SARS-CoV-2 ingresa a cuidados intensivos. De éstos, de 29 a 75\% requiere ventilación mecánica invasiva con una mortalidad asociada de 12 a $81 \%$. El síndrome de insuficiencia respiratoria aguda (ARDS, por sus siglas en inglés) es la forma de presentación más grave. La fisiopatología del ARDS secundario al SARS-CoV-2 difiere de las causas convencionales. Presenta desregulación en la vasoconstricción pulmonar hipóxica, hipertensión pulmonar aguda secundaria y fenómenos microtrombóticos. El desarrollo de hipoxemia grave refractaria $\left(\mathrm{PaO}_{2}<60 \mathrm{mmHg}\right.$ o $\mathrm{PaO}_{2} / \mathrm{FiO}_{2}<100 \mathrm{mmHg}$, con $\mathrm{FiO}_{2}$ de 80 a $100 \%$, un PEEP $>10$ a $20 \mathrm{cmH}_{2} \mathrm{O}$ por al menos de 10 a 12 horas) constituye el escenario de máxima gravedad con una mortalidad asociada de 71 a $94 \%$. El uso de estrategias de rescate que impacten en la fisiopatología específica de esta entidad como el uso de óxido nítrico inhalado (ONi), el bloqueo neuromuscular y la ventilación en prono han surgido como objetivos terapéuticos de interés. La ventilación mecánica protectora (presión meseta [Pmes] $<27 \mathrm{cmH}_{2} \mathrm{O}$ y presión conducción [PC] $<15$ $\mathrm{cmH}_{2} \mathrm{O}$ ) continúa siendo la piedra angular de su manejo. Objetivos: Determinar si existe asociación entre el uso de óxido nítrico inhalado y la ventilación en prono prolongado con la mecánica ventilatoria en pacientes con hipoxemia grave refractaria secundaria a infección por SARS-CoV-2. Material y métodos: Se realizó un estudio de cohorte histórica, retrospectivo, descriptivo, comparativo y retrolectivo. Se analizaron los datos de los expedientes de pacientes ingresados a la UCI Respiratoria del Centro Médico ABC con diagnóstico de ARDS secundaria a infección por SARS-CoV-2 que requirieron óxido nítrico inhalado y ventilación mecánica en prono del $1^{\circ}$ de abril al 31 de diciembre de 2020. Se realizó un análisis univariado, el análisis estadístico se realizó en SPSS versión 21, se analizaron medidas de tendencia y dispersión, así como análisis de los factores de riesgo con prueba t de Student y $\chi^{2}$. Resultados: Se analizaron un total de 108 pacientes de los cuales 54 recibieron ONi. Conclusiones: El uso de ONi en pacientes con hipoxemia grave refractaria a ventilación en prono y bloqueo neuromuscular no produjo una mejoría estadísticamente significativa en la oxigenación; sin embargo, permitió reprogramar el soporte ventilatorio para lograr mantener al paciente en metas de protección alveolar.

Delta de ácido úrico como predictor de severidad en pacientes con SARS-CoV-2

Pérez Fernández Oyuky, Mendoza Portillo Elizabeth,

Mendoza Rodríguez Martín

Hospital General La Villa, SSACDMX.

Antecedentes: EI SARS-CoV-2 representa la primera causa de mortalidad actual en la población mexicana, a nivel global se han acumulado 1'919,126 defunciones. Las regiones con más muertes son América (47\%) y Europa (33\%). Hasta la semana 2 del año
2021 en México se estimaba 1'541,633 casos, siendo la Ciudad de México la entidad más afectada; hasta ese momento se habían registrado 18,443 defunciones, con una necesidad hospitalaria de $80 \%$ y con alta demanda de hospitalización en el servicio de unidad de cuidados intensivos (UCI). Por esta razón, surge la necesidad de nuevos marcadores tempranos de severidad y pronóstico. Objetivo: Correlacionar el delta de ácido úrico (DAU) con la mortalidad en pacientes con SARS-CoV-2 tratados en la UCI, así como su relación con otras variables de severidad. Material y métodos: Se realizó un estudio longitudinal en la UCI con 71 pacientes; las variables de estudio fueron: demográficas, comorbilidades, días de estancia en la $\mathrm{UCI}$, días de ventilación mecánica (VM), escala predictora de mortalidad, ácido úrico inicial y ácido úrico final; desenlace: mortalidad. Resultados: De los participantes, 69\% fueron varones, 31\% mujeres, la edad promedio fue de 54.35 años ( \pm 10.28 ), ácido úrico al ingreso de $3.9 \mathrm{mg} / \mathrm{dL}$ ( \pm 1.74$)$, ácido úrico al egreso de 2.89 $\mathrm{mg} / \mathrm{dL}( \pm 1.70)$, delta de ácido úrico promedio de $1.077 \mathrm{mg} / \mathrm{dL}( \pm$ $1.59 \mathrm{mg} / \mathrm{dL}$ ), Apache II al ingreso de 18.35 puntos ( \pm 9.04$)$ al egreso de 22.95 puntos $( \pm 19.68)$, mortalidad global de $50.7 \%$, de los cuales $78.67 \%$ requirió ventilación mecánica y $21.12 \%$ no requirió. La correlación de Spearman para DAU y mortalidad fue $r=-0.34$, el índice de determinación r2 $=0.13$, con significancia $p<0.004$, (IC) $95 \%$. Conclusiones: Se demostró la correlación entre el DAU con la mortalidad de los pacientes con SARS-CoV-2 con adecuada significancia estadística a un intervalo de corte de 1.0-1.5 mg/dL, y una mortalidad de $50.7 \%$. Adicionalmente, se demostró que dicho intervalo tuvo correlación con el inicio de la ventilación mecánica. Se identificó que sí existe relación entre la puntuación de Apache II y la mortalidad por SARS-CoV-2; para este estudio un puntaje mayor a 18 demostró la mejor significancia estadística.

\section{Protección pulmonar durante la fase proliferativa}

de SDRA por SARS-CoV-2, asociada a

supervivencia, en un hospital de tercer nivel

Cruz Islas Esteban Alejandro, González Pérez Netzahualcóyotl,

De La Cruz Salome Roseli, Maynez Rojas Daniel Jesús,

López Carrillo Lilia

Centro Médico ISSEMyM Toluca.

Las metas de protección pulmonar, presión meseta, driving pressure se asocian a supervivencia. Metodología: Se realizó un estudio observacional, retrospectivo, longitudinal, comparativo, en pacientes con SARS-CoV-2 con ventilación mecánica. Se calculó la supervivencia en pacientes que se encontraban en metas de protección pulmonar y aquéllos en los que no se encontraban en las mismas, durante los 14 días posteriores al ingreso a la unidad de cuidados intensivos, se estimó la supervivencia a 28 días de cada una de ellas de manera individual, para esto se utilizó el método Kaplan-Meier, mediante la prueba Log-rank se identificó si existía diferencia significativa de la supervivencia entre grupos. Resultados: Se reclutaron 130 pacientes con diagnóstico de SARS-CoV-2. Con una supervivencia global a 28 días de $69.2 \%$. La presión máxima se asoció a supervivencia en $64.2 \%$ de estos días, la presión meseta en $71.4 \%$, driving pressure en $64.2 \%$, con una $p<0.05$, no así el volumen tidal, el cual en ninguno de los días se asoció a supervivencia de manera estadísticamente significativa. Conclusión: La presión máxima $\leq 35 \mathrm{cmH}_{2} 0$, presión meseta $\leq 30 \mathrm{cmH}_{2} \mathrm{O}$, driving pressure $\leq 15 \mathrm{cmH}_{2} \mathrm{O}$ se asoció a una mayor supervivencia a 28 días, a diferencia del volumen tidal entre 6-8 $\mathrm{mL} / \mathrm{kg}$ de peso predicho.

Mortalidad en pacientes SARS-CoV-2 con traqueostomía percutánea en la unidad de cuidados intensivos de un hospital centinela Rosales León Jorge Roberto, González Pérez Netzahualcóyotl Centro Médico ISSEMYM Toluca.

Introducción: Uno de los beneficios de una traqueostomía incluye menor utilización de sedantes, adecuada higiene pulmonar, además 
facilita el retiro de la ventilación mecánica. La traqueostomía percutánea es un procedimiento de rutina realizado en nuestra unidad de cuidados intensivos; sin embargo, no existe información en nuestro país acerca de la evolución de los pacientes con SARS-CoV-2 a quienes se les somete a este procedimiento en la unidad de cuidados intensivos. Material y métodos: Se realizó un estudio observacional, retrospectivo, longitudinal y descriptivo, en pacientes con SARS-CoV-2 que requirieron ventilación mecánica prolongada y se les realizó traqueostomía percutánea. Se obtuvieron datos demográficos, día de realización de la traqueostomía, días libres de ventilación mecánica, desarrollo de neumonía asociada a ventilación mecánica y muerte durante su estancia en la unidad de cuidados intensivos. Resultados: Se analizaron un total de 52 pacientes de 28-71 años, con una media de edad de 47 años (DE $=9.86)$, con un total de 42 pacientes masculinos y 10 pacientes femeninos representando $80.7 \%$ y $19.2 \%$, respectivamente. El día de la realización de la traqueostomía fue en promedio a los 22 días, el día más temprano que se realizó la traqueostomía fue a los 14 días en un total de cuatro pacientes $(7.6 \%)$ y el día de realización más tardío fue a los 28 días en dos pacientes (3.8\%). El 5.7\% de los pacientes presentaron complicaciones tempranas. El número de pacientes que desarrollaron neumonía asociada a la ventilación mecánica fue un total de 39 pacientes (75\%); un total de 19 pacientes murieron, lo que representa $36.54 \%$, de los cuales cinco fueron femeninos y 14 masculinos. Los datos obtenidos arrojaron una media de días de muerte de $15.37 \pm 2$ posterior a la traqueostomía. Conclusión: La realización de traqueostomía percutánea en pacientes COVID-19 en la unidad de cuidados intensivos del centro médico ISSEMYM Toluca, se realiza en un promedio a los 22 días de ventilación mecánica, con la mínima incidencia de complicaciones, un porcentaje importante $(75 \%)$ de los pacientes desarrolló neumonía asociada a la ventilación mecánica y la mortalidad fue alta (36.5\%).

Síndrome coronario agudo con elevación del

ST en el contexto de choque séptico

Rivera Chávez Manuel José, Valero Vidal Fernanda,

Urzúa González Agustín Ramiro,

Galván Martínez Nathalia, Nieto Saucedo JR

Hospital Regional de Alta Especialidad del Bajío. León, Guanajuato, México.

Introducción: El infarto al miocardio (IAM) tipo II se define como un IAM secundario a isquemia por un incremento en la demanda de oxígeno o un descenso en su aporte sanguíneo coronario. Entre los hallazgos más frecuentemente encontrados en el ECG de pacientes con sepsis y choque séptico se encuentran: prolongación del intervalo QTc, ondas $\mathrm{J}$ de Osborn, bloqueos de rama, cambios no específicos en el segmento ST y, más raramente, elevación del segmento ST sugestiva de infarto al miocardio. Objetivo: Evaluar la elevación de biomarcadores cardiacos y del segmento ST en sepsis o choque séptico. Material y métodos: Paciente femenino de 53 años con síndrome coronario agudo con elevación del segmento ST elevado. Resultados: Paciente con elevación del segmento ST en contexto de choque séptico de origen abdominal, pulmonar y urinario. Discusión: El mecanismo de elevación de biomarcadores cardiacos y del segmento ST en sepsis o choque séptico es multifactorial. Puede deberse a hipoperfusión tisular, así como a la diseminación de la infección al tejido cardiaco.

ICU-STORM: incidencia de síndrome de liberación de citocinas en COVID-19 en la unidad de cuidados intensivos adultos Sarabia Collazo Ángel Antonio, González Ponce Carlos Mauricio, Sánchez Nava Víctor Manuel, Chávez Pérez Carlos Eduardo,

Razcón Echeagaray Arturo

Hospital San José, Tecnológico de Monterrey.

Los pacientes con COVID-19 presentan un espectro clínico que va desde una infección asintomática hasta la falla respiratoria secundaria a síndrome de distrés respiratorio del adulto que culmina en falla orgánica múltiple. Debido a que el virus SARS-CoV-2 tiene la capacidad de activar el sistema inmune de forma desproporcionada a la lesión y puede desencadenar una liberación importante de citocinas, denominado «tormenta de citocinas» o «síndrome de liberación de citocinas», resulta razonable dar seguimiento a los niveles séricos de IL-6 y utilizar herramientas diagnósticas como el H-score para detectar y diagnosticar estados proinflamatorios y/o de inmunocompromiso. Se investigó la incidencia del síndrome de liberación de citocinas en 130 pacientes ingresados a terapia intensiva del Hospital San José en Monterrey, Nuevo León, México, de predominio masculino (71\%), edad media de 57 años y una puntuación media SOFA de 8.29 al ingreso a la UCIA. EI H-Score fue de 64.35 puntos como valor medio, encontrando una baja incidencia en la población estudiada. Debido a estos valores no se pudo establecer correlación entre H-score con SOFA, días de estancia hospitalaria, días de ventilación mecánica y mortalidad. A pesar de que el H-Score tiene una sensibilidad de $93 \%$ y especificidad de $86 \%$ para diagnosticar linfohistiocitosis hemofagocítica $(\mathrm{HLH})$, no se encontró utilidad en el abordaje de pacientes con COVID-19; sin embargo, los valores séricos de IL-6 presentan una relación directamente proporcional con estancia hospitalaria y mortalidad $(p=0.001)$, lo que respalda el concepto de tormenta de citocinas y sugiere que una población de estudio con mayor incidencia de HLH es necesaria para establecer la significancia estadística apropiada.

Estrategias ventilatorias en síndrome de distrés respiratorio agudo por COVID-19 en la Unidad de Cuidados Intensivos de la Unidad Médica de Alta Especialidad Hospital de Especialidades No. 1 IMSS. León, Guanajuato, en el periodo del $1^{\circ}$ de abril al 31 de octubre del 2020

Ortega Roy José Armando, Fernández Hernández Juan Pablo, Bravo Santibáñez Edgar, Hernández González Martha Alicia,

Contreras Chávez Marisol

CMN del Bajío, León, Guanajuato.

En diciembre de 2019 se identificó un nuevo coronavirus, llamado SARS-CoV-2, en Wuhan, China. Actualmente, el virus SARS-CoV-2 es el causante de una nueva epidemia global denominada enfermedad por coronavirus 2019 (COVID-19), la cual tiene afectación principalmente pulmonar y puede llegar a presentar síndrome de distrés respiratorio agudo. Se ha demostrado que la mortalidad es elevada, aún más en nuestro país en pacientes con SDRA por COVID-19 en comparación a la mortalidad que se tiene en países europeos. Desde el año 2020 a la fecha se han propuesto diferentes fenotipos pulmonares en los que se puede manifestar el SDRA por COVID-19 y en cada fenotipo pulmonar se recomiendan estrategias ventilatorias diferentes para mejorar los índices de oxigenación y reducir la mortalidad; sin embargo, siguen existiendo discrepancias entre los fenotipos propuestos y el manejo ventilatorio para cada uno; por ello la importancia de determinar las estrategias ventilatorias implementadas en nuestra unidad de cuidados intensivos y comparar con las estrategias ventilatorias internacionales, ya que tienen un impacto en la mortalidad. Objetivo: Describir las estrategias ventilatorias en el síndrome de distrés respiratorio agudo por COVID-19 en la unidad de cuidados intensivos de la Unidad Médica de Alta Especialidad del Hospital de Especialidades No.1, Centro Médico del Bajío León, Guanajuato, en el periodo del $1^{\circ}$ de abril al 31 de octubre de 2020. Material y métodos: Se realizó un estudio ambispectivo, descriptivo, en el periodo comprendido del $1^{\circ}$ de abril al 31 de octubre de 2020, se incluyeron todos los pacientes confirmados de COVID-19, ya sea por pruebas séricas o RT-PCR que cumplan con criterios diagnósticos de SDRA por COVID-19 que ingresaron a la unidad de cuidados intensivos de la Unidad Médica de Alta Especialidad del Hospital de Especialidades No.1, Centro Médico del Bajío León, Guanajuato. Se registró el modo ventilatorio utilizado y los parámetros ventilatorios empleados en los pacientes a su ingreso a la unidad de cuidados intensivos y cada 24 horas hasta las $96 \mathrm{~h}$, así como el registro de los pacientes que se les realizó posición prono y se determinó si fueron o no respon- 
dedores; se asignó el fenotipo pulmonar correspondiente según los criterios de la literatura internacional; las variables cualitativas como pronación, motivo de egreso, sexo, se presentaron como frecuencia de proporciones. Resultados: Se incluyeron 76 casos consecutivos de pacientes hospitalizados en la unidad de cuidados intensivos con diagnóstico de SDRA por COVID-19. La mediana (IRQ) de edad de los pacientes fue de 56 años (41.7-61.25 años). El fenotipo $\mathrm{H}$ fue el más frecuente en $75 \%$ de los casos $(n=57)$. Dentro de los parámetros ventilatorios que se registraron y midieron al ingreso se observó una mediana de PEEP de 9 (8-10), una mediana de $\mathrm{FiO}_{2}$ de 65 (entre 53.7-80) y una relación $\mathrm{PaO}_{2} / \mathrm{FiO}_{2}$ con una mediana de 88.35 (70-100). Los parámetros ventilatorios medidos y registrados a las 96 horas fueron un $\mathrm{FiO}_{2}$ con una mediana de 40 (35-50), PEEP de 8 (8-9) y una relación $\mathrm{PaO}_{2} / \mathrm{FiO}_{2}$ con una mediana de 190 (136-217.8). La maniobra de prono se realizó en $90.79 \%$ de los casos $(n=69)$, de los cuales se consideraron respondedores $84.2 \%(n=64)$, con una mediana de duración de tres días de pronación (2-3). Los días de estancia en la unidad de cuidados intensivos fue una mediana de 11 (8-14), con una mediana de días de ventilación mecánica de 10 (713). Se observó una mortalidad de $27.6 \%(n=21)$. Conclusión: En las estrategias ventilatorias en síndrome de distrés respiratorio agudo por COVID-19 al interior de la unidad de cuidados intensivos de la Unidad Médica de Alta Especialidad del Hospital de Especialidades No.1, Centro Médico del Bajío León, Guanajuato, se encontró la utilización de PEEP en $9 \mathrm{cmH}_{2} \mathrm{O}$ a comparación del uso de PEEP mayor a $12 \mathrm{cmH}_{2} \mathrm{O}$ en el fenotipo $\mathrm{H}$, de igual forma se observó la pronación temprana, ajuste de PEEP conforme a metas de protección pulmonar, complianza y relación $\mathrm{PaO}_{2} / \mathrm{FiO}_{2}$. Esto tuvo un impacto que mejoró la supervivencia en estos pacientes y se tuvo una mortalidad menor a lo que se menciona en la literatura internacional en pacientes con síndrome de distrés respiratorio agudo por COVID-19.

\section{Respuesta inmune e inmunosupresión en pacientes con ventilación mecánica por infección por SARS-CoV-2 \\ Ramírez Urízar Diego Andrés, Aisa Álvarez Alfredo, \\ Aguirre Janet Silvia, Chaires Gutiérrez Rodrigo, \\ Bórquez López Yazmín Fabiola, Coronado Robles Celia Margarita Centro Médico ABC.}

Introducción: La pandemia de SARS-CoV-2 ha inspirado intriga sobre la respuesta inmune a dicho virus, especialmente en paciente graves con síndrome de distrés respiratorio del adulto (SDRA). Este estudio describe el comportamiento de la respuesta inmune, inmunosupresión y sus desenlaces en los pacientes con ventilación mecánica (VM). Material y métodos: Cohorte prospectiva. Del 23 de marzo al 31 de diciembre de 2020 se recolectó información basal, parámetros ventilatorios, gasométricos y estudios de laboratorio de todos los pacientes mayores de 18 años con COVID-19 que recibieron VM, con registros hasta el día 15 de VM. Se dividieron los grupos en pacientes vivos a los 90 días y defunciones. Resultados: Registramos 218 pacientes, con mortalidad de $23 \%$. En el día 1 de VM los pacientes no presentaron diferencias en conteos celulares o reactantes de fase aguda, excepto dímero D 1,020 (705-1,711) vs 1,328 (940-2,340) ng/ $\mathrm{dL}, \mathrm{p}=0.035$. En el análisis de regresión lineal de efectos mixtos se observan diferencias cronológicas estadísticamente significativas en leucocitos y proteína $\mathrm{C}$ reactiva (PCR) concordante con la elevación de la presión de distensión alveolar (PDalv). No se encontró asociación con mortalidad en el uso de tocilizumab 2.20 (0.279-17.358) y corticosteroides 0.54 (0.229-1.273) en riesgos proporcionales de COX al día 1 de VM. Durante los 15 días de VM los pacientes que fallecieron recibieron dosis más altas de corticosteroides, dosis a partir 150 $\mathrm{mg} / \mathrm{día}$ equivalente a prednisona se asocian a mortalidad. Conclusiones: Existe evolución cronológica similar en elevación de PCR, leucocitos y elevación PDalv, explicada por la disminución de la distensibilidad pulmonar estática (Cstat) y presión positiva al final de la espiración total (PEEPtotal). El uso de tocilizumab no tuvo asociación con la mortalidad y las dosis equivalentes a prednisona entre 100-150 $\mathrm{mg} / \mathrm{día}$ se asocian a mejores resultados.
Delta de $\mathrm{CO}_{2}$ como predictor de lesión renal aguda (LRA) en pacientes con diagnóstico de síndrome de distrés respiratorio del adulto (SDRA) por COVID-19 y choque séptico

Villalobos Álvarez Víctor Hugo, Sánchez Calzada Armando,

Arcos Zamora Magally, Varela Sánchez Luis Ernesto,

Aguirre Sánchez Janet Silvia; Chaires Gutiérrez Rodrigo

Centro Médico ABC.

Introducción: El virus SARS-CoV-2 es capaz de afectar diversos órganos llevándolos a disfunción. Los principales órganos de choque son el pulmón, riñón y sistema cardiovascular. Los pacientes que desarrollan SRDA suelen presentarse con sepsis y choque séptico, siendo más propensos a desarrollar lesión renal aguda (LRA), ya sea por hipovolemia o disfunción miocárdica. El monitoreo invasivo en estos pacientes ha resultado todo un reto, debido a los protocolos sanitarios y la gran cantidad de pacientes, por lo que la diferencia de $\mathrm{CO}_{2}$ arterio-venosa $\left(\mathrm{DCO}_{2}\right)$, que es un parámetro fácil de medir, nos puede ayudar a determinar de forma indirecta el gasto cardiaco (GC) y perfusión en pacientes con choque. Objetivo: Evaluar el uso del $\mathrm{DCO}_{2}$ como un predictor de LRA en pacientes con choque séptico y COVID-19. Material y métodos: Se realizó un estudio observacional, transversal y retrolectivo en pacientes con choque séptico y SDRA por COVID-19, ingresados en la unidad de cuidados intensivos (UCI) respiratorios del Centro Médico $A B C$. Se determinó el $\mathrm{DCO}_{2}$ y su asociación con LRA. Se resumieron los datos usando medidas de tendencia central, $t$ de Student para determinar diferencia de medias y se estimó el riesgo para desarrollar LRA calculando odds ratio (OR). El estudio se aprobó por el comité de ética del Centro Médico ABC, Ciudad de México (Folio: ABC-21-36). Resultados: Desde el 13 de marzo al 13 de julio del 2020 se admitieron 527 pacientes (p) con diagnóstico de COVID-19, de los cuales 107 (20.3\%) presentaron SRDA con requerimiento de ventilación mecánica invasiva (VMI) y 99 (18.78\%) choque séptico. El $74.4 \%$ fueron hombres, el $61 \%$ desarroIló algún grado de LRA, sobre todo en el grupo con $\mathrm{DCO}_{2}$ menor a 6 $\mathrm{mmHg}$ (44 vs $17 \mathrm{p} \leq 0.001)$ con OR 2.108 , IC $95 \%=1.23-3.36$. No hubo diferencia significativa en las escalas de gravedad. Conclusión: $\mathrm{DCO}_{2}$ mayor de $6 \mathrm{mmHg}$ no fue buen predictor para LRA; sin embargo, un $\mathrm{DCO}_{2}$ menor a $6 \mathrm{mmHg}$ aumentó el riesgo de LRA.

\section{Eventos trombóticos y marcadores inflamatorios en} pacientes con neumonía grave por síndrome respiratorio agudo severo coronavirus-2 (SARS-CoV-2)

López Reymundo Paulo Sergio, Rodríguez Santos Ahtziri Yunuén, Palacios Chavarría Adrián, Álvarez Alfredo Aisa,

Aguirre Sánchez Janet Silvia, Chaires Gutiérrez Rodrigo Centro Médico ABC.

Introducción: La neumonía por SARS-CoV-2 se asocia a secreción importante de citoquinas y aglomeramiento de células inmunológicas, que activan las células endoteliales condicionando coagulopatía, afectando al pulmón en una fase temprana, con un fenotipo clínico del Síndrome de Dificultad Respiratoria Aguda (SDRA), que posteriormente progresa a respuesta inflamatoria sistémica desregulada por marcadores inflamatorios que causan mayor lesión endotelial generando trombosis. Muchos pacientes presentan niveles elevados de Dímero $D$ (DD), así como de Proteína-C-Reactiva (PCR), Interleucina-6 (IL-6) y ferritina, supeditando la formación de coágulos, con la interrupción de la circulación (arterial o venosa) a cualquier nivel del sistema circulatorio. Objetivos: Determinar si existe relación entre los niveles elevados de marcadores inflamatorios y eventos trombóticos en pacientes con neumonía por SARS-CoV-2 con Ventilación Mecánica Invasiva (VMI). Material y métodos: Realizamos estudio de una cohorte, observacional, retrospectivo y longitudinal, en pacientes que ingresaron a la Unidad de Terapia Respiratoria del Centro Médico ABC, de abril a julio de 2020, con diagnóstico de neumonía por SARS-CoV-2 con VMI. Utilizamos el programa STATA para el análisis estadístico. Efectuamos un análisis lineal de medidas repetitivas por regresión logística para evaluar el comportamiento cronológico de las variables inflamatorias. 
Posteriormente, ajustamos los marcadores inflamatorios con variables demográficas, para la obtención de certeza diagnóstica y predicción de riesgo de eventos trombóticos. Resultados: Analizamos 100 pacientes, predominando el sexo masculino con $78 \%$. El 18\% presentó trombosis. Inicialmente los marcadores inflamatorios estadísticamente significativos fueron DD $(p=0.010)$ con niveles de $1375.5 \mathrm{ng} / \mathrm{mL}(967-$ $2651)$ y ferritina $(p=0.030)$ con niveles $1391.5 \mathrm{ng} / \mathrm{mL}(622-1779)$. Con el ajuste de variables inflamatorias por edad, género, índice de masa corporal (IMC) y escalas de gravedad, las variables estadísticamente significativas fueron DD $(p=0.001)$ y ferritina $(p=0.004)$, obteniendo certeza diagnóstica de $80.57 \%$ para predecir el riesgo de eventos trombóticos. Conclusión: El control estricto de los parámetros de laboratorio y un alto índice de sospecha son vitales para formular un enfoque de tratamiento personalizado para los pacientes, y también pueden ayudar a clasificar a los pacientes con alto riesgo de presentar eventos trombóticos. Nuestro modelo enfatiza que hay que tener precaución con niveles elevados de DD y ferritina.

\section{Comparación de interleucina 6, ferritina, dímero}

$D$ y deshidrogenasa láctica como predictores de mortalidad en pacientes con SARS-CoV-2

Castillo-Amador Jovanhy, Álvarez-Calderón Jovanhy, Uribe-Sánchez Roberto Salvador, Chanona-Toalá Eduardo Antonio, Alabarda Pérez Alejandra Montserrat, Rodríguez García José Neftalí Hospital Regional 1ㅇ de Octubre, ISSSTE.

Introducción: La pandemia causada por el nuevo síndrome respiratorio agudo severo coronavirus 2 (SARS-CoV-2) presenta un enorme desafío para la salud pública en el mundo. Se han sugerido diversos biomarcadores relacionados con la gravedad de la enfermedad y complicaciones, como marcadores pronósticos, entre ellos el dímero D, IL-6, LDH y ferritina. Material y métodos: Estudio observacional, analítico y retrospectivo en el que se analizaron datos clínicos y variables de interés de 64 expedientes de pacientes ingresados a la unidad de cuidados intensivos, en el periodo junio a diciembre del 2020, con un rango de edad entre 18 y 85 años. Como estadística se empleará estadística descriptiva (porcentaje, desviación estándar, promedios) para las variables cualitativas chi cuadrada. Los datos se analizaron con SPSS 22. Resultados: Se incluyeron 64 pacientes en el presente estudio, de los cuales, $72 \%$ fueron del sexo masculino, registrándose una mayor mortalidad en éstos en comparación al sexo femenino, con una mortalidad general de $52 \%$. En cuanto a los niveles bioquímicos de IL-6, dímero D y ferritina al ingreso de los pacientes con SARS-CoV-2 no se encontró una significancia estadística en relación a su desenlace, siendo la $\mathrm{LDH}>300 \mathrm{u} / \mathrm{L}$ la única con diferencia estadística significativa que se asoció con una mayor mortalidad $(p=0.006)$. Conclusiones: $\mathrm{Se}$ observó una mayor asociación entre los niveles elevados de LDH y mortalidad, incluso con un corte menor a lo reportado en la literatura, siendo éste el único en comparación con IL-6, ferritina y dímero $\mathrm{D}$, con un peso estadístico significativo y con un menor costo para su realización.

\section{La hipoxemia como factor de riesgo para}

lesión renal aguda en COVID-19

Betancourt Vera Jesús Emmanuel, Peniche Moguel Karla Gabriela, Sánchez Díaz Jesús Salvador, De la Cruz García Rocha María Guadalupe, Calyeca Sánchez María Verónica

Hospital de Especialidades No. 14, CMN «Adolfo Ruiz

Cortines", IMSS. Veracruz, Veracruz.

Introducción: La lesión renal aguda (LRA) es la pérdida rápida de la función renal, la neumonía con insuficiencia respiratoria aguda (IRA) es la forma más grave de COVID-19, la hipoxemia puede ser un determinante para LRA. Material y métodos: Estudio de cohorte, retrospectivo, descriptivo, analítico, se incluyeron pacientes en el servicio de urgencias del $1^{\circ}$ de mayo al 30 de septiembre de 2020 con la definición de «caso sospechoso de COVID-19» más imágenes sugestivas de COVID-19 por tomografía. Se clasificaron por el valor de creatinina sérica ( $\mathrm{CrS}$ ) de ingreso: Grupo 1 sin LRA y Grupo 2 con LRA; se buscó la asociación entre la hipoxemia y LRA. Resultados: Se incluyeron 105 pacientes. La mediana de $\mathrm{CrS}$ al ingreso fue 0.7 $\mathrm{mg} / \mathrm{dL}$ y $1.0 \mathrm{mg} / \mathrm{dL}$ para el $\mathrm{G} 1$ y $\mathrm{G} 2$, respectivamente $(p=0.05)$. La mediana de $\mathrm{PaO}_{2} / \mathrm{FiO}_{2}$ para el $\mathrm{G} 1$ fue de $90 \mathrm{mmHg}$ y $\mathrm{G} 2$ de 105 $\mathrm{mmHg} p=0.76$, asociación entre LRA e hipoxemia con Pearson: 0 , la saturación arterial de oxígeno $\left(\mathrm{SatO}_{2}\right) \geq 92 \%$ presentó correlación negativa para LRA (Pearson: $-0.537 \mathrm{p}=0.04$ ). Conclusión: La hipoxemia no es un factor desencadenante para LRA en COVID-19, la optimización de la oxigenoterapia y monitoreo con la $\mathrm{SatO}_{2}$ deberían intensificarse desde el ingreso al servicio de urgencias.

\section{Asociación y valor predictivo del índice poder}

mecánico/ distensibilidad pulmonar con días

libres de ventilación mecánica en pacientes con

COVID-19 en una unidad de terapia intensiva

Turrubiates Hernández Theno Alexandro,

Ramírez Gutiérrez Álvaro Eduardo, Sánchez Medina Jorge Rosendo, Reyes Pérez Amalia Angélica, Morales Hernández José Fernando,

Azua Guevara Reyna Isabel

Hospital Regional Cd. Madero, Petróleos Mexicanos.

Introducción: El poder mecánico (PM) se asocia a mortalidad y se debe ajustar a las características pulmonares en pacientes COVID-19. Material y métodos: Estudio observacional, retrospectivo, analítico de septiembre 2020 a febrero 2021. Parámetros de ventilación y análisis de la curva ROC y modelos de regresión de Poisson para OR. El objetivo primario fue determinar la asociación entre el índice PM/ Cest y días libres de ventilación mecánica (DLVM). Resultados: Se incluyeron 43 pacientes. Se dividió en sobrevivientes $(n=25,58.1 \%)$ y no sobrevivientes $(n=18,41.9 \%)$. Se eligió mediana de DLVM con los parámetros de VMI. El índice PM/Cest inicial ABC 0.73 (IC 95\% [0.58-0.88], $p=0.008$ ), punto de corte de $0.90 \mathrm{~J} / \mathrm{min} / \mathrm{mL} / \mathrm{cm} \mathrm{H}_{2} \mathrm{O}$. La regresión multivariable de Poisson el índice $\mathrm{PM} / \mathrm{Cest}$ inicial fue de OR 0.18 (IC 95\% [0.03-0.98], p = 0.047). Discusión: Coppola documentó a pacientes con SDRA no COVID-19, el valor de PM absoluto no demostró significancia; al ajustarlo a Cest fue independiente para mortalidad. Conclusiones: El índice PM/Cest al inicio se asoció de forma significativa e independiente con DLVM en COVID-19. Punto de corte de $0.9 \mathrm{~J} / \mathrm{min} / \mathrm{mL} / \mathrm{cmH} 20$ con mejor sensibilidad y especificidad para predecir los DLVM y esto podría reducir la mortalidad.

Decúbito prono prolongado: una estrategia no ventilatoria en neumonía grave por SARS-CoV-2

Vargas García Irvin Jair, Sánchez Díaz Jesús Salvador, Peniche Moguel Karla Gabriela,

González Escudero Eduardo Alberto,

García García Víctor Daniel, Calyeca Sánchez María Verónica

Hospital de Especialidades No. 14, CMN «Adolfo Ruiz

Cortines", IMSS. Veracruz, Veracruz.

Introducción: La neumonía grave por SARS-CoV-2 (Severe Acute Respiratory Syndrome Coronavirus 2) ocasiona hipoxemia severa, por lo anterior, las guías para el manejo de pacientes adultos críticamente enfermos con COVID-19, recomiendan el uso de la posición decúbito prono para mejorar la oxigenación. Material y métodos: Estudio de cohorte, prospectivo, descriptivo y analítico. Pacientes ingresados a la unidad de cuidados intensivos en el periodo comprendido entre el 18 de abril del 2020 al 18 de agosto del 2021 con VMI secundaria a neumonía grave por SARS-CoV-2 confirmados. Resultados: En el periodo comprendido se incluyeron 110 pacientes que cumplieron con los criterios inclusión. Del total, 88 pacientes se incluyeron en el grupo de MEJORÍA SOSTENIDA AL RETIRO DEL PRONO y 22 en el grupo de MEJORÍA NO SOSTENIDA AL RETIRO DEL PRONO. Se observó que el porcentaje de disminución de la $\mathrm{PaO}_{2} / \mathrm{FiO}_{2}$ al retiro del prono es útil para predecir mortalidad con ABC de 0.740 con IC $95 \%$ de $(0.646-0.834)$ y $p=0.001$. Conclusión: La disminución $>50 \%$ 
de la $\mathrm{PaO}_{2} / \mathrm{FiO}_{2}$ al retiro de la posición decúbito prono prolongado o mejoría no sostenida es un predictor de mortalidad en los pacientes con neumonía grave por SARS-CoV-2.

\author{
Índices de oxigenación: comparando el pentágono \\ de la difusión alveolar como predictores de \\ mortalidad en pacientes con COVID-19 \\ Duran Pimentel Enid Georgina, Ramírez Salas Nery Denisse, \\ Cortés Román Jorge Samuel, Rivera Solís Gerardo, \\ Villegas Domínguez Josué Eli \\ Hospital Regional ISSSTE, Veracruz.
}

Introducción: Para mejorar la oxigenación, puede ser útil tener un índice para determinar la naturaleza de la deficiencia que está causando la insuficiencia respiratoria. Clínicamente, esta información ayudaría a hacer el diagnóstico inicial y podría ayudar a decidir si el paciente se beneficiaría de la admisión a la $\mathrm{UCl}$ en función de la probabilidad de revertir con éxito la afección. Material y métodos: Se realizó un estudio de cohorte prospectivo, se ingresaron todos los pacientes con diagnóstico de infección por SARS-CoV-2 con prueba confirmada por PCR, en un periodo de tiempo comprendido del $1^{\circ}$ marzo a septiembre de 2021 que ingresaron a unidad de cuidados intensivos, donde se correlacionó a los índices de oxigenación con el incremento de la mortalidad. Resultados: La población estudiada se compuso de 58 pacientes. La $\mathrm{PAO}_{2}$, se observó en la media al ingreso, de los pacientes que fallecieron se obtuvo una media 456.25 \pm 156.61 ( $p=0.5$ ), se encontró que a las 72 horas de ingreso en los pacientes que permanecían con una $\mathrm{PAO}_{2}$ mayor de 426.87 se tenía un factor de riesgo para mortalidad con una $p=0.01$. Encontramos que la $\mathrm{paO}_{2} / \mathrm{PAO}_{2}$ al ingreso de los pacientes que fallecieron presenta una media $0.44 \pm 0.39(p=0.3)$, a las 72 horas una media de $0.24 \pm$ $0.13(p=0.1)$. Con respecto a la A-aDO2 se obtuvo que los pacientes que mantenían una media de $355.27 \pm 182.88$ presentaban mayor riesgo de mortalidad con una $p=0.02$. En cuanto al Índice respiratorio, se obtuvo una media al ingreso de los pacientes que fallecieron de $5.24 \pm 2.96(p=0.1)$. Conclusión: A diferencia de lo reportado en la población mundial en nuestro estudio, no encontramos diferencia o asociación significativa de los índices de oxigenación a su ingreso en $\mathrm{UCl}$ como predictor de mortalidad.

Poder mecánico como predictor de mortalidad en los pacientes con diagnóstico de neumonía por SARSCoV-2 que recibieron ventilación mecánica invasiva Avendaño González Luis Mario, Morán Nares Luis Ángel, Cortés Román Jorge Samuel, Huanca Pacaje Juan Marcelo, Guízar Miguel Martín Alejandro, Cortés Guillén Abisai Hospital Regional de Alta Especialidad, ISSSTE, Veracruz.

Introducción: Las fuerzas mecánicas generadas durante la ventilación mecánica por la interacción entre el ventilador y el sistema respiratorio pueden dañar al pulmón en un proceso que se ha denominado lesión inducida por el ventilador. El grado de lesión se ha relacionado con la cantidad de energía transferida desde el ventilador mecánico al sistema respiratorio dentro de un periodo de tiempo determinado, denominado poder mecánico, datos experimentales basados en tomografías sugieren que el poder mecánico mayor de $12 \mathrm{~J} / \mathrm{min}$ podría generar lesión. Se proyecta como otra de las variables a controlar dentro de las estrategias de protección pulmonar, determinado en estudios experimentales como un umbral de energía a partir del cual inician los cambios mecánicos en el pulmón que pueden conducir a lesión inducida por el ventilador. Material y métodos: Se realizó un estudio retrospectivo, analítico, comparativo, se ingresaron todos los pacientes con diagnóstico de neumonía por SARS-CoV-2 que requirieron ventilación mecánica invasiva, en un periodo de tiempo comprendido de marzo-agosto del 2021 que ingresaron a unidad de cuidados intensivos y utilizaron el poder mecánico como variable para predecir mortalidad. Resultados: La población estudiada se compuso de 67 pacientes; se evaluó la asociación entre el poder mecánico alto a las 48 horas y la mortalidad, se documentó que $49.25 \%(N=33)$ de los pacientes que mantuvieron el poder mecánico alto en 48 horas murieron; $28.35 \%(\mathrm{~N}=19)$ con poder mecánico alto no se asoció con mortalidad, se realizó una prueba de asociación con $\chi^{2}$ cuadrada de Pearson en el que se obtiene un valor de $p=0.105$, por lo que no existe diferencia estadísticamente significativa y no se corrobora la asociación entre la mortalidad de los pacientes con poder mecánico alto (> $12 \mathrm{~J} / \mathrm{min}$ ) a las 48 horas. Conclusión: El poder mecánico puede considerarse como otra variable a controlar como estrategia de protección pulmonar del paciente con infección por SARS-CoV-2, basado en que la energía transmitida al pulmón tiene mayor impacto en los pacientes que reciben ventilación mecánica por un intervalo de tiempo mayor de 7 días, con un promedio de estancia en la unidad de cuidados intensivos $12.3 \pm 6.2$ días y el promedio de días de ventilación mecánica invasiva $9.2 \pm 5.6$.

Medición de la vaina del nervio óptico mediante tomografía computarizada como predictor de mortalidad en pacientes con traumatismo craneoencefálico grave al ingreso a la unidad de cuidados intensivos

Martínez Romero Karen Saraí, Cantú Cárdenas Héctor Alberto, Ramírez Barba Christián, Borbolla González Adán de Jesús, Gutiérrez Martínez Carlos Alberto

Hospital de Especialidades, Centro Médico Nacional de Occidente IMSS.

Antecedentes: El traumatismo craneoencefálico (TCE) es una condición médico-quirúrgica caracterizada por una afectación cerebral secundaria a una lesión de tipo traumática. Los pacientes con TCE grave tienen un alto riesgo de mortalidad y ésta va a depender de diferentes factores como la presencia de hipertensión intracraneal, la edad, origen de la lesión y puntuación en la escala de coma de Glasgow. La medición del diámetro de la vaina del nervio óptico (DVNO) parece ser un buen indicador indirecto de hipertensión intracraneal, y por consiguiente un buen predictor de mortalidad. Objetivo general: Determinar el punto de corte más adecuado, así como la utilidad de la medición del DVNO como indicador pronóstico de mortalidad en los pacientes con TCE grave en la unidad de cuidados intensivos (UCI). Resultados: Se estudiaron 60 expedientes de pacientes con TCE que ingresaron a UCl, 51 eran hombres (85\%), 45 pacientes sobrevivieron $(75 \%)$ y 15 pacientes fallecieron $(25 \%)$. La edad promedio fue de $50.5 \pm 10.6$ años, el puntaje Glasgow promedio al ingreso fue de $6.6 \pm 1.6$ puntos, el IMC promedio fue de $26.42 \pm 4.10 \mathrm{~kg} / \mathrm{m}^{2}$ y el número de días promedio de estancia en $\mathrm{UCl}$ fue de $9.03 \pm 6.4$. El diámetro del nervio óptico no fue un predictor de mortalidad, pero sí lo fue la escala de coma de Glasgow con un ABC de 0.775 (IC95\%, 0.648-0.901, $p=0.002$ ), el mejor punto de corte fue de 7 con una sensibilidad de $93 \%$ y una especificidad de $54 \%$. El modelo de regresión lineal bivariada señala al puntaje de coma de Glasgow bajo y la estancia hospitalaria extensa como predictores de mortalidad. Conclusiones: Los resultados de este estudio infieren que, en congruencia con la evidencia científica actual, las características sociodemográficas de nuestra población son similares a las reportadas por otros autores, siendo los hombres aproximadamente de 50 años los más afectados por esta entidad. Por otra parte, la medición del diámetro de la vaina del nervio óptico ha sido considerada un buen indicador pronóstico de hipertensión intracraneal, que a su vez se asocia con el aumento de la mortalidad. Sin embargo, en el presente estudio no existe una asociación entre el diámetro de la vaina del nervio óptico y el pronóstico de mortalidad.

\section{Exactitud diagnóstica del índice de nocicepción analgesia para la evaluación del dolor en pacientes críticos Cinco Huiqui Abraham Ismael, Beltrán Moguel Jonathan, Trejo Arteaga Alejandro, Cerón Díaz Ulises Wilfrido Hospital Español.}

La evaluación de la presencia de dolor en el enfermo sometido a sedación profunda y ventilación mecánica es posible a través de escalas 
que se basan en datos clínicos indirectos. El índice de nocicepción analgesia (ANI) es utilizado en enfermos anestesiados en la sala quirúrgica. La información sobre su eficacia en los enfermos críticos es escasa. Este trabajo prospectivo, observacional y analítico evalúa el rendimiento diagnóstico de ANI para detectar dolor en los enfermos críticos en ventilación mecánica, sedados con RASS de -3 a -1. Como estándar, se utilizaron dos escalas clínicas (BPS y CPOT) aplicadas por médicos y enfermeras de manera simultánea a la medición de ANI, en cuatro ocasiones durante un periodo de 12 minutos. En 23 enfermos y 224 mediciones, la capacidad discriminativa medida a través de áreas por debajo de curvas ROC osciló entre 0.779 y 0.817 . El puntaje de ANI con mejor capacidad diagnóstica osciló entre 52 y 55. Utilizando el valor umbral de 50 , la sensibilidad osciló entre 0.418 y 0.524 , la especificidad entre 0.910 y 0.980 . Se reportan los valores predictivos y las razones de verosimilitud. Los cambios en ANI y las escalas, originados por procedimientos durante la medición, tuvieron una concordancia que osciló entre 28 y $41 \%$. Los resultados identifican una tecnología con potencial para su utilización en el enfermo crítico. Son necesarios más estudios que corroboren nuestros resultados.

Índices de oxigenación, índice de choque diastólico y tiempo en presión soporte como predictores de éxito en el retiro de la ventilación mecánica invasiva en paciente críticamente enfermo con COVID-19 Palacios Morales Juan Carlos, Soni Aguilera Víctor, Huanca Pacaje Juan Marcelo, Cortés Román Jorge Samuel, Rivera Solís Gerardo, Villegas Domínguez Josué Eli Hospital Regional de Alta Especialidad, ISSSTE, Veracruz.

Introducción: El porcentaje de pacientes gravemente enfermos por COVID-19 que toleran el retiro de la ventilación mecánica es mínimo, por lo cual es fundamental determinar cuáles son los predictores de éxito en el retiro de la ventilación mecánica. Objetivos: Determinar si los índices de oxigenación, tiempo en presión soporte e índice de choque diastólico son predictores de éxito en el retiro de la ventilación mecánica en pacientes críticamente enfermos por COVID-19. Material y métodos: Estudio de cohorte prospectivo en pacientes con COVID-19 que ingresaron a la unidad de cuidados intensivos y fueron sometidos a pruebas de ventilación espontánea, siendo posteriormente liberados del ventilador mecánico. Se recolectaron datos demográficos, resultados de laboratorio y parámetros ventilatorios con la finalidad de analizar y determinar predictores de éxito en el retiro de la ventilación mecánica. Resultados: Se observó una diferencia significativa entre los pacientes que toleraron y los que no el retiro de la ventilación mecánica en las siguientes variables: índice de respiración rápida superficial $47 \pm 17.9$ vs $90 \pm 20.2$ (valor de $p$
$=0.08$ ), días de ventilación mecánica 5.8 vs 9.3 (valor de $p=0.03$ ), sesiones de prono 1.36 vs 2.33 (valor de $p=0.058$ ) y presión arterial diastólica $57 \pm 3.6$ (valor de $p=0.027$ ). Conclusión: En los pacientes críticamente enfermos por COVID-19 que son candidatos a retiro de la ventilación mecánica, sugerimos usar como predictores de éxito el índice de respiraciones rápidas superficiales $<64$, días de ventilación mecánica $<8$, presión arterial diastólica $>57 \mathrm{mmHg}$ y haber tenido máximo 2 sesiones de prono como predictores de éxito en el retiro de la ventilación mecánica.

Evaluación de la escala modificada simplificada de riesgo nutricional (mNUTRIC) en pacientes ingresados en la unidad de cuidados intensivos por neumonía por SARSCoV-2 y comparación con la evaluación de falla orgánica secuencial (SOFA) en el desenlace clínico de los pacientes Méndez Reyes Raquel, Marín Romero María del Carmen, Santos González Brenda, Chanona Toalá Eduardo Antonio, Marroquín Barrera Manuel Alejandro

Hospital Regional 1ํ de Octubre, ISSSTE, CDMX, México.

Introducción: Diversos puntajes clínicos de severidad de la enfermedad y pronóstico de mortalidad son usados de manera rutinaria en la práctica clínica, la escala mNUTRIC demostró que cada incremento de 1 punto en mNUTRIC se asoció a un riesgo 1.4 veces mayor de mortalidad a 28 días en pacientes ingresados por otras patologías. El uso de esta herramienta se ha valido también en población con infección por SARS-CoV-2. Material y métodos: Se realizó un paquete estadístico IBM $^{\odot}$ SPSS $^{\odot}$ Statistics versión 22 calculando sensibilidad, especificidad, valor predictivo positivo, valor predictivo negativo y realizando curva COR a 64 pacientes de 18 a 85 años de edad, ingresados a la unidad de cuidados intensivos del Hospital Regional $1^{\circ}$ de Octubre del ISSSTE por neumonía por SARS-CoV-2 en el periodo del $1^{\circ}$ de junio al 31 de diciembre del 2020. Resultados: Se encontró para el sistema mNUTRIC score modificado una sensibilidad de $51.6 \%$ y una especificidad de $45.2 \%$, con valor predictivo positivo $48.42 \%$, valor predictivo negativo $48.29 \%$; el sistema SOFA mostró una sensibilidad de $29 \%$ y especificidad de $67.7 \%$, con valor predictivo positivo 47.38 y valor predictivo negativo $48.81 \%$. Para determinar la exactitud diagnóstica de estos test, se elaboró una curva COR, con una significancia de 0.757 y 0.778 , respectivamente (IC 95\%). Conclusiones: No se encontraron diferencias entre SOFA y mNUTRIC score, pero hay una tendencia hacia mNUTRIC en ser mejor predictor de mortalidad que SOFA. Sin embargo, con respecto a la sensibilidad y especificidad, ambas están por debajo de $60 \%$, por lo que no puede optarse por una de ellas por sí solas como predictores de mortalidad en neumonía por SARS-CoV-2. 